\title{
Avaliação dos Efeitos de uma Modalidade de Treinamento de Habilidades Sociais para Crianças
}

\author{
Evaluation of the Effects of the Social Skills Training Method to Children
}

\author{
Elaine Sabino Gonçalves*1 \& Sheila Giardini Murta \\ Universidade Católica de Goiás, Goiânia, Brasil
}

\begin{abstract}
Resumo
Este artigo descreve os efeitos de uma intervenção em habilidades sociais sobre comportamentos pró-sociais, autoconceito e aceitação pelos pares, em três crianças: P.1, comportamento agressivo e dificuldade de aprendizagem, gênero feminino, 9 anos; P.2, agressividade, gênero masculino, 7 anos; P.3, timidez excessiva, dificuldade de aprendizagem, gênero masculino, 13 anos. O programa foi baseado em técnicas cognitivocomportamentais e foi feito em grupo, em 20 sessões. A avaliação de resultados, feita a partir do relato das crianças, de seus pais e pares, evidenciou um aumento em comportamentos pró-sociais e mudanças positivas no autoconceito e no julgamento dos pares. Recomenda-se que programas futuros voltados para crianças com dificuldades de aprendizagem estendam o treinamento em habilidades sociais para o contexto escolar.

Palavras-chave: Habilidades sociais; agressividade infantil; timidez; intervenção em grupo.
\end{abstract}

\begin{abstract}
This article describes the effects of a social skills training on prosocial behavior, self concept, and peers acceptance, in three children: P.1, female, 9 years old, aggressive behavior and difficulty of learning; P.2, male, 7 years old, aggressive behavior; P.3, male, 13 years old, extremely shy, difficulty in learning, The program was cognitive behavioral based and was conducted in group, in 20 sessions. The evaluation of the results done through children, peers and parents' reports showed an increase in prosocial behavior and positive changes in self concept and peers judgment. Future programs to children with learning difficulties should expand the social skills training to the school environment.

Keywords: Social skills; child aggressiveness; shyness; group intervention.
\end{abstract}

Atualmente, os profissionais da área de saúde mental tem se voltado para o desenvolvimento de tratamentos eficazes para os transtornos emocionais e comportamentais em crianças e adolescentes. Isto se deve a alta incidência de problemas experimentados por crianças e jovens e a posterior seqüência de disfunções tais como: fraco desempenho acadêmico, delinqüência, abuso de drogas, crises conjugais e desordens emocionais variadas (Del Prette \& Del Prette, 2001b).

Há evidências de que se a criança desenvolver um amplo repertório de habilidades sociais terá mais probabilidade de estabelecer, futuramente, relações sociais mais saudáveis e com menor risco de rejeição por seus pares (Castro, Melo, \& Silvares, 2003; Ladd, Herald, Slutzky\& Andrews, 2004). Além desse fato, estudos sugerem que o desenvolvimento de habilidades sociais na infância pode se constituir em um fator de proteção contra a ocorrência de dificuldades de aprendizagem e de comportamentos anti-sociais

\footnotetext{
* Endereço para correspondência: Rua Alameda dos Eucaliptos, Q. 01, L. 14, Parque dos Eucaliptos, Anápolis, GO, 75083405. Tel.: (62) 33184410 e 8438 2722. E-mail: elainesagles@hotmail.com

${ }^{1}$ Este artigo descreve parte da experiência da primeira autora no Estágio em Psicologia Clínica, feito no Centro de Estudos, Pesquisa e Prática Psicológica da Universidade Católica de Goiás, sob a orientação da segunda autora.
}

(Baraldi \& Silvares, 2003; Fariz, Mias, \& Moura, 2005; Koch \& Gross, 2005; Marinho, 2003).

Tal fator de proteção pode ser estabelecido à medida que comportar-se com habilidades sociais favorece a obtenção de reforçadores sociais importantes como amizade, respeito, status no grupo ou, genericamente, em convivência cotidiana mais agradável. Para isto, Del Prette e Del Prette (2005) cita as seguintes classes de habilidades sociais relevantes na infância: autocontrole e expressividade emocional, habilidades de civilidade, empatia, assertividade, solução de problemas interpessoais, fazer amizades e habilidades sociais acadêmicas. Com efeito, muitos estudos mostram que a competência social na infância, termo que reflete julgamento social sobre a qualidade geral do desempenho individual em uma determinada situação, apresenta correlação positiva com vários indicadores de funcionamento adaptativo como rendimento acadêmico, responsabilidade, independência e cooperação (Bandeira, Rocha, Souza, Del Prette, \& Del Prette, 2006; Caballo, 1996; Marinho \& Caballo, 2002).

Assim, um repertório social empobrecido pode constituir em um sintoma ou correlato de problemas psicológicos, podendo se expressar como dificuldades interpessoais na infância. Tais dificuldades podem ser classificadas, na psicopatologia infantil, em dois grandes grupos de com- 
portamento: os externalizantes (que se expressam predominantemente em relação a outras pessoas) e os internalizantes (que se expressam predominantemente em relação ao próprio indivíduo) (Del Prette \& Del Prette, 2005). Ambas as classes de comportamento podem ser associadas aos conceitos de excesso e déficit comportamental. Comportamentos internalizantes são evidenciados por retraimento, depressão, ansiedade e queixas somáticas. Em contraposição, comportamentos externalizantes são marcados por impulsividade, agressão, agitação, características desafiantes e anti-sociais. Assim, os dois grupos de comportamento dificultam o desenvolvimento psicossocial da criança, já que os internalizantes podem privar a criança de interagir com o ambiente, isto é, o indivíduo pode evitar iniciar uma interação com pares ou adultos e, por outro lado, os externalizantes podem gerar conflitos e provocar rejeição de pais, professores e colegas (Bolsoni-Silva, Marturano, Pereira, \& Manfrinato, 2006; Gomide, 2003; Marinho, 2003; Patterson, Reid, \& Dishion, 2002).

Portanto, o sucesso das etapas formativas do indivíduo depende, primordialmente, do processo de socialização. É, inicialmente, no contato com os pais que a criança aprende uma série de habilidades motoras, lingüísticas e afetivas, necessárias para a orientação em seu ambiente físico e social. Todo esse repertório passará por contínua transformação, em decorrência do ingresso em novos grupos sociais que reforçarão comportamentos adequados e inadequados socialmente (Bolsoni-Silva \& Marturano, 2002; Gomide, 2003).

Devido a este fator, Löhr (2003), apresenta um programa desenvolvido pela equipe da universidade federal do Paraná, direcionado a um grupo de crianças entre 4 a 6 anos e que integra o atendimento dos pais. A intervenção consistiu em 10 encontros grupais, com 90 minutos de duração e intervalos semanais. Cada grupo continha em média 6 crianças. Tanto para a s crianças quanto para os pais, os temas abordados foram assertividade, solução de problemas, leitura do contexto e empatia. Porém, no grupo de crianças, foram feitas atividades lúdicas, enquanto o grupo de pais recebeu instruções acerca de como prover condições antecedentes e reforçadoras de comportamentos socialmente habilidosos em seus filhos. Orientações semelhantes àquelas fornecidas aos pais foram fornecidas para os professores das crianças participantes do programa, a cada 15 dias. Uma sessão individual para devolução dos resultados aos pais e encaminhamento foi feita ao fim da intervenção. Os resultados foram avaliados através de observação direta do comportamento das crianças, com uso de registro de evento e amostragem de tempo, feitas durante atividades escolares e durante a sessão terapêutica e de respostas dos pais ao Child Behavior Check-list de Achenbach (Achenbach, 1966, citado em Löhr, 2003), antes e após a intervenção. Os relatos dos pais evidenciaram progressos no repertório de habilidades sociais dos filhos, todavia não confirmados através dos dados observacionais obtidos durante as atividades escolares e sessões terapêuticas. Foram discutidas sugestões para aprimoramento dos procedimentos de avaliação e cuidados para maximizar a adesão ao programa. Em função das desistências ocorridas ao longo do programa, a autora advertiu que os resultados não foram generalizados, já que apenas pais altamente motivados permaneceram até o fim da intervenção.

Marinho (2005), propôs um programa de intervenção comportamental em grupo para pais de crianças e préadolescentes e as crianças, com idades entre 3 a 11 anos. $\mathrm{O}$ programa teve como meta ensinar aos pais a observar e descrever o comportamento da criança e a serem agentes mais eficazes de reforço, aumentando a freqüência, a variedade e a extensão das recompensas sociais para a criança e reduzindo a freqüência de comportamentos verbais competidores, como ordens, críticas e questionamentos. Os pais também foram ensinados a lidar com condutas manipuladoras dos filhos. A intervenção junto ao grupo de crianças teve como objetivo desenvolver comportamentos pró-sociais através do treinamento em habilidades sociais. Os resultados demonstraram que o programa produziu mudanças comportamentais positivas na maioria das famílias submetidas à intervenção. A avaliação foi realizada através da aplicação do CBCL, observação direta na sala de espera do padrão da interação pais-filhos e registros do comportamento dos filhos.

A análise destes estudos anteriores mostra diferentes possibilidades de estratégias de avaliação de programas de habilidades sociais. Medidas psicométricas, observacionais e verbais podem ser utilizadas, a partir de múltiplos informantes, como a própria criança, seus pais, professores, pares e terapeutas (Murta, 2005). Esta tendência é produtiva para o avanço dos conhecimentos na área, tendo em vista a abrangência dos dados coletados. Neste sentido, o presente artigo descreve uma experiência de intervenção com um grupo de crianças com problemas internalizantes e externalizantes e uma avaliação a partir de relatos de múltiplos informantes: os próprios participantes, seus pais e pares. Especificamente, este artigo visa descrever uma intervenção em habilidades sociais para crianças e seus efeitos sobre o aumento de comportamentos pró-sociais, mudanças em autoconceito e no julgamento de pares.

\section{Método}

\section{Participantes}

Os participantes foram selecionados a partir das fichas de triagem do Centro de Estudos, Pesquisa e Prática Psicológica (CEPSI), a clínica-escola de Psicologia da Universidade Católica de Goiás. Foram buscadas fichas de crianças cujos pais se queixavam de condutas agressivas em seus filhos. Além deste critério, para a inserção da criança no grupo, os pais teriam que participar do grupo de treinamento em práticas educativas parentais e habilidades sociais educativas (Coelho \& Murta, 2005).

Participaram do programa seis crianças, quatro meninos e duas meninas, entre sete a 13 anos de idade, com nível socioeconômico baixo, cursando desde a terceira à quinta série do ensino fundamental. Elas foram subdivididas em dois grupos, vespertino e noturno, segundo a disponibilidade de horário dos mesmos e os respectivos pais. Contudo, este artigo irá enfocar apenas o grupo vespertino (ver Tabela 1). 
Psicologia: Reflexão e Crítica, 21(3), 430-436.

Tabela 1

Descrição dos Participantes do Programa

\begin{tabular}{|c|c|c|c|c|}
\hline Participantes & Comportamento-problema & Gênero & Idade & Série \\
\hline P. 1 & Agressividade e dificuldade de aprendizagem & Feminino & 9 & $3^{\mathrm{a}}$ \\
\hline P.2 & Agressividade & Masculino & 7 & $3^{\mathrm{a}}$ \\
\hline P.3 & Timidez excessiva e dificuldade de aprendizagem & Masculino & 13 & $5^{\mathrm{a}}$ \\
\hline
\end{tabular}

\section{Instrumentos}

Foram usados os instrumentos: Escala de Percepção do Autoconceito Infantil (PAI), Ficha para Avaliação Sociométrica e Inventário de Comportamentos da Infância e Adolescência (CBCL).

A Escala de Percepção do Autoconceito Infantil (PAI) é de autoria de Sánchez e Escribano (1999) e consta de 34 itens com figuras representando determinadas situações na escola, na família, e outros contextos de intervenção que avaliam a autonomia, relações sociais da criança, agressividade e estados afetivos. Há duas opções para cada item, representativas de um autoconceito positivo ou negativo, e a criança deve escolher aquela com a qual mais se identifica.

A Ficha de Avaliação Sociométrica, elaborada pelas autoras, foi usada para a realização da avaliação sociométrica, que consiste na coleta de informações sobre os tipos e a qualidade do relacionamento social de uma pessoa com seu grupo de companheiros. Foi solicitado por escrito aos colegas de classe dos participantes, relatar o nome de um a três colegas da escola de quem ela mais gostava ou menos gostava para estudar, brincar ou participar de alguma atividade. Com base na quantidade de escolhas que cada criança recebia, era computado o escore de preferência social ou rejeição. As informações obtidas permitem identificar o status social da criança em termos de aceitaçãorejeição pelos pares, e classificá-las em populares (com predominância de votos de aceitação), rejeitadas (com predominância de votos de rejeição), negligenciadas (sem votos de aceitação rejeição), controversas (com votos de aceitação e rejeição em proporções similares) e medianas (votos de aceitação e rejeição próximos ao escore do grupo) (Coie, Doodge, \& Copotelli, 1982, citados em Castro et al., 2003).

O Inventário de Comportamentos da Infância e Adolescência (CBCL), de autoria de Achenbach (1991), é um questionário que avalia competência social e problemas de comportamento em crianças e adolescentes de quatro a 18 anos, a partir de informações fornecidas pelos pais. Neste trabalho foi aplicada somente a primeira parte desse instrumento, que corresponde à avaliação da competência social, agrupada em três escalas: atividade, sociabilidade e escolaridade, fornecendo a soma dos escores brutos das três escalas o escore final da competência social. Os resultados ponderados no CBCL permitem classificar as crianças em: não-clínica, limítrofe e clínica (Bordin, Mari, \& Caiero, 1995). Para a escala de competência social, a categoria clínica corresponde a escores abaixo de 30 , a limítrofe entre 30 e 33 e a categoria não-clínica acima de 33 .
Local

O programa foi realizado em um consultório do CEPSI usado para atendimento a grupos de crianças, equipado com mesa, cadeiras, espelho, lousa, almofadas e brinquedos. Além deste mobiliário, foram utilizados os materiais: jogo terapêutico para pais e filhos (Moura, 2005), jogos lúdicos, artigos de papelaria tais como papel e lápis, filmadora e fitas VHS.

\section{Procedimento}

O programa para as crianças foi realizado em 20 sessões, de 90 minutos cada e periodicidade semanal. O primeiro contato com as crianças foi realizado individualmente, com o objetivo de estabelecer vínculo, conhecer as queixas das crianças, esclarecer dúvidas acerca do trabalho da psicologia, além de verificar a disponibilidade e motivação destas frente à participação no grupo. A segunda e a penúltima sessão foram destinadas à avaliação pré e pós-teste, com aplicação da Escala de Percepção do Autoconceito Infantil nas crianças. A última sessão foi destinada ao fornecimento de feedback, avaliação dos colegas, comemoração das melhoras obtidas e encerramento do trabalho. As demais sessões foram planejadas de acordo com os temas relacionados a habilidades sociais, tais como comunicação eficaz, relacionamento interpessoal, empatia, lidar com as emoções, tomada de decisão e resolução de problemas (Del Prette\& Del Prette, 2001a, 2001b, 2005; Stallard, 2004). Os temas e técnicas usados no programa estão sumarizados na Tabela 2. Duas sessões com os pais, ao começo e ao final do programa, foram feitas para a aplicação da Lista de Verificação Comportamental para Crianças e Adolescentes.

Foi utilizado um Termo de Consentimento Livre e Esclarecido, solicitando autorização aos pais para filmagem de uma sessão, e uma carta à escola onde as crianças estudavam, solicitando autorização para aplicação da Ficha para Avaliação Sociométrica.

\section{Resultados}

Os resultados foram analisados a partir da Escala de Percepção do Autoconceito Infantil (PAI), da Ficha de Avaliação Sociométrica e da Lista de Verificação Comportamental pra Crianças e Adolescentes (CBCL).

A Escala de Percepção do Autoconceito Infantil evidenciou um aumento nos escores relativo à percepção positiva dos participantes (ver Tabela 3). Nos quesitos relativos à agressividade e ao relacionamento interpessoal as mudan- 
Gonçalves, E. S. \& Murta, S. G. (2008). Avaliação dos Efeitos de uma Modalidade de Treinamento de Habilidades Sociais para Crianças.

ças foram acentuadas, pois no pré-teste as respostas que indicavam o comportamento inadequado de briga e isolamento foram assinaladas. Já na avaliação pós-teste a iden- tificação com comportamentos mais habilidosos socialmente foram pontuados.

Tabela 2

Procedimento das Sessões do Programa de Habilidades Sociais com Crianças

\section{Sessão Tema Objetivo Técnica}

1 Contato inicial

2 Contrato e avaliação pré-teste

3 Tomada de decisão

4 Auto-conhecimento

5 Solução de problemas

6 Empatia

7 Importância de regras

8 Habilidades sociais assertivas

9 Compartilhar emoções

10 Trabalhar em grupo

11 Dar e receber feedback

12 Relacionamento entre pais e filhos

13 Reconhecer sentimentos

14 Auto-estima

15 Briga

16 Expressar raiva e pedir mudança de comportamento

17 Falar em público

18 Analisar pedidos recebidos

19 Avaliação pós-teste

20 Encerramento
Discutir sobre a função da clínica, da terapia e do psicólogo. Preparação para o grupo

Integrar-se com os outros membros do grupo. Decidir em conjunto os termos para o funcionamento do grupo.

Analisar e decidir conseqüências, riscos e benefícios que uma situação pode apresentar.

Promover conhecimento e aceitação que cada indivíduo tem de si mesmo (qualidades e limitações)

Desenvolver capacidade de enfrentar as situações problemáticas de forma construtiva

Desenvolver habilidade de compreender o outro

Analisar causa-conseqüência das regras, percebendo a necessidade das mesmas

Conversar com pessoas de autoridade. Desenvolver argumentação e justificativas. Aceitar crítica e alterar o próprio desempenho

Nomear emoções e reconhecê-las no outro

Manifestar opinião, concordar, discordar

Descrever o desempenho de uma pessoa. Tarefa de casa para férias

Observar diretamente a interação entre pais e filhos

Nomear e perceber o sentimento do outro.

Reconhecer as próprias qualidades e limitações

Discutir comportamentos inadequados como brigas

Expressar adequadamente sentimentos negativos de raiva, desagrado e desgosto

Desenvolver a fluência verbal e a criatividade

Fazer, aceitar e recusar pedidos

Reavaliar auto-conceito das crianças

Avaliar a satisfação com o programa e encerrá-lo
Sessão individual, exposição dialogada

Apresentação do crachá. Aplicação da Escala de Percepção do

Autoconceito Infantil

Ensaio comportamental

Desenho do corpo

Semáforo (Stallard, 2004)

Vivendo o papel do outro (Del Prette \& Del Prette, 2001a)

Desenho grupal da escola sem regras

Entrada no céu (Del Prette \& Del Prette, 2001a)

Boneco de borracha

Formando um grupo (Del Prette

\& Del Prette, 2001a)

Fazer as malas. Diário

Jogo terapêutico para pais e filhos (Moura, 2005)

Discussão do diário. Cor dos sentimentos (Del Prette \& Del Prette, 2005)

Desenho e comemoração

Livros de estórias infantis

Ensaio comportamental

História coletiva oral (Del Prette \& Del Prette, 2001a)

Peça o que quiser (Del Prette \& Del Prette, 2001a)

Aplicação da Escala de Percepção do Autoconceito Infantil e jogos lúdicos

Comemoração. 
Tabela 3

Escores da Escala de Percepção do Autoconceito Infantil

\begin{tabular}{lcc}
\hline Participantes & $\begin{array}{l}\text { Escores do } \\
\text { pré-teste* }\end{array}$ & $\begin{array}{l}\text { Escores do } \\
\text { pós-teste* }\end{array}$ \\
\hline P.1 & 57 & 65 \\
P.2 & 52 & 60 \\
P.3 & 63 & 66 \\
\hline
\end{tabular}

Nota. * A pontuação máxima possível de ser obtida é 68 pontos.

Os resultados da Ficha de Avaliação Sociométrica apontaram mudanças positivas na percepção dos pares. $\mathrm{O}$ índice de aceitação e rejeição obtidos na primeira e segunda avaliação demonstraram que: P.1, anteriormente classificado como rejeitado, não obteve nenhuma rejeição pelos colegas de classe ao final do programa passando para a categoria negligenciada; P.2 aumentou o escore de aceitação e passou a fazer parte da categoria mediana; P.3, classificada como criança negligenciada ao início do programa, conseguiu duas aceitações no pós-teste, escore semelhante ao que maioria dos colegas de classe obtiveram. A Tabela 4 descreve os resultados da Ficha de Avaliação Sociométrica.

Tabela 4

Dados da Avaliação Sociométrica Pré-Teste e Pós-Teste

\begin{tabular}{lcccc}
\hline \multirow{2}{*}{$\begin{array}{c}\text { Participantes } \\
\text { Aceitação }\end{array}$} & \multicolumn{2}{c}{ Rejeição } & \multicolumn{2}{c}{ Pós-teste } \\
& Aceitação & Rejeição \\
\hline P.1* & 1 & 7 & 1 & 0 \\
P.2* & 1 & 2 & 3 & 0 \\
P.3* & 0 & 0 & 2 & 0 \\
\hline
\end{tabular}

Nota. * P.1 foi avaliado por um grupo de 25 crianças, * P.2 por 16 crianças e * P.3 por 21.

Os resultados do CBCL demonstraram que a percepção dos pais mudou em relação ao comportamento dos filhos, pois foi evidenciado a predominância da categoria clínica (fraco desempenho em competência social e problemas de comportamento) ao início do programa e não clínico ao final do mesmo (participantes P.2 e P.3), demonstrando um aumento significativo no escore da competência social. $\mathrm{O}$ participante P.1 foi categorizado como não-clínico antes da intervenção, mantendo-se nesta categoria ao final do programa com escore positivo aumentado.

Tabela 5

Escores do CBCL Pré-Teste e Pós-Teste

\begin{tabular}{lrlrl}
\hline & \multicolumn{2}{c}{ Pré-teste } & \multicolumn{2}{c}{ Pós-teste } \\
Participantes & Escores & Categoria & Escores & Categoria \\
\hline P.1 & 34 & Não-clínica & 36 & Não-Clínica \\
P.2 & 29 & Clínica & 35 & Não-clínica \\
P.3 & 18 & Clínica & 39 & Não-clínica \\
\hline
\end{tabular}

\section{Discussão}

Os resultados encontrados apontam para um aumento de comportamentos pró-sociais e estão em acordo com estudos similares (Baraldi \& Silvares, 2003; Marinho, 2005). Segundo Baraldi e Silvares (2003), o treino de habilidades sociais infantis e treino comportamental dos pais promove uma melhor relação entre pais e filhos. Contudo, este mesmo estudo destaca o fato de que não houve mudança na percepção positiva dos pares frente à criança atendida. Tal fato entra em desacordo com o presente estudo, pois se constatou mudanças na percepção dos pares em relação às crianças atendidas ao fim da intervenção.

Uma importante evidência da efetividade do programa desenvolvido foi obtida através do uso de diferentes medidas de avaliação e múltiplos informantes, avaliando a percepção das próprias crianças em relação a si, com a Escala de Percepção do Autoconceito Infantil; a percepção dos pares, com a Ficha de Avaliação Sociométrica; e dos pais frente aos respectivos filhos por meio do CBCL. Todos estes instrumentos apontaram melhoras no comportamento das crianças e evidenciam consistentemente o aumento de comportamentos pró-sociais.

Segundo a Escala de Percepção do Autoconceito Infantil, P.1 e P.2 que apresentavam e se identificavam com comportamentos agressivos, passaram a ter uma percepção positiva frente a situações de brigas e convívio social e P.3, caracterizado como uma criança tímida, apresentou-se participante dos grupos e questões que também favoreciam o convívio social. De acordo com Castro et al. (2003), o êxito ou o fracasso percebidos nas relações impactam a autoavaliação, reforçando (positiva ou negativamente) determinadas condutas. Assim, os resultados da escala demonstraram um considerável aumento no escore de aceitação deles próprios, indicando um possível aumento no repertório que possui comportamentos assertivos sobre o meio como: autonomia, estabelecimento de relacionamentos interpessoais, confiança em si próprio, segurança e desempenho satisfatório em atividades variadas como esportes e estudos.

Coerentemente com os dados citados acima, a Ficha de Avaliação Sociométrica retratou mudanças na percepção dos pares frente às crianças atendidas. Ao início da intervenção, o participante P.1 obteve um alto índice de rejeição pelos colegas, demonstrando déficits em várias habilidades sociais de civilidade e de atendimento a normas de convivência social, porém no pós-teste não apresentou nenhuma rejeição pelo grupo. Já o participante P.3, categorizado como uma criança negligenciada pelo pouco envolvimento com colegas e a baixa assertividade e expressividade emocional, conseguiu ao final do treinamento se aproximar da categoria mediana. Tais resultados apontaram para uma provável diminuição de comportamentos coercitivos para com os pares, pois os participantes que haviam recebido alguma rejeição (P.1 e P.2) no início do programa não obtiveram nenhuma indicação ao final. Além deste fator, os participantes P.2 e P.3 aumentaram a aceitação nos respectivos grupo de pares, refletindo o aumento de comportamentos pró-sociais. 
Outro fator que merece destaque são os resultados apontados pelo CBCL, que apontaram a passagem das crianças da categoria clínica para não-clínica. Estes dados sinalizaram o melhor desempenho em competência social e problemas de comportamento, ocorridos com os participantes P.2 e P.3 após a intervenção. Além dos dados do CBCL, os filhos relataram durante as sessões finais como estavam percebendo as mudanças nas práticas educativas de seus pais. O participante P.3 relatou que "antes minha mãe era uma onça, não podia chegar perto", tal fala demonstra a diferenciação do comportamento da mãe, que punia a criança apenas pelo estado de humor ou dava atenção somente quando a criança se comportava de uma forma inadequada. Segundo Patterson et al. (2002), os pais de crianças anti-sociais falharam em reforçar os comportamentos prósociais dos filhos e em estabelecer limites frente a comportamentos agressivos, prejudicando desta forma o desenvolvimento de um repertório de habilidades sociais. Por esta razão, o atendimento a crianças e seus respectivos pais tem sido padrão nos demais programas realizados na área de agressividade infantil e habilidades sociais, pois os pais precisam aprender a se comportarem de uma maneira adequada e contingente ao comportamento dos filhos.

Os resultados deste estudo mostraram que os pais (Coelho \& Murta, 2007) e o grupo de pares tem um papel fundamental no desenvolvimento de habilidades sociais. P.1 já havia apresentado comportamentos inadequados como roubo e mentiras excessivas. Contudo tais comportamentos passaram a serem punidos negativamente (retirada do reforço) e supervisionados pelos pais, e estes passaram também a reforçar comportamentos adequados que aconteciam com pouca frequiência. Dessa forma, comportamentos pró-sociais passaram a ocorrer com uma freqüência maior.

Uma das fragilidades desta intervenção é que as dificuldades de aprendizagem das crianças não foram alvo direto da intervenção. Considerando que tais dificuldades estavam presentes entre as queixas dos pais, teria sido pertinente o planejamento e implementação de ações com fins avaliativos ou de intervenção focadas na aprendizagem na escola. Programas futuros poderão estender suas ações à escola e incluir intervenções interdisciplinares, envolvendo psicólogos e psicopedagogos, e poderão incluir também a avaliação de desempenho acadêmico.

Esta experiência de intervenção mostrou-se bem sucedida em alcançar seus objetivos práticos: os participantes apresentaram melhoria em seu auto-conceito e em seu desempenho social, conforme sua própria avaliação e a avaliação de seus pais e colegas de classe. Estes resultados promissores justificam a replicação desta intervenção junto a outros grupos. Sugere-se uma avaliação mais abrangente deste programa, com a associação entre medidas verbais (Del Prette \& Del Prette, 2005) e observacionais. Em uma das sessões da intervenção, foi feita uma filmagem da interação entre os pais e filhos em uma sessão de jogo. Este procedimento mostrou-se extremamente rico para se observar diretamente a responsividade parental. A observação dos pais em situação de jogo terapêutico (Moura,
2005) poderá ser usada no futuro, como medida de pré e pós-teste em estudos na área. Sugere-se ainda a observação, durante as sessões, da emissão de comportamentos socialmente habilidosos pelas crianças. Espera-se que o uso de múltiplas medidas e múltiplos informantes possa contribuir para o crescimento desta área de pesquisa no Brasil e refinar a qualidade das intervenções oferecidas a crianças e adolescentes.

\section{Referências}

Achenbach, T. M. (1991). Manual for the child behavior checklist/ 4-18 and 1991 profile. Burlington, VT: University of Vermont.

Bandeira, M., Rocha, S. S., Souza, T. M. P., Del Prette, Z. A. P., \& Del Prette, A. (2006). Comportamentos problemáticos em estudantes do ensino fundamental: Características da ocorrência e relação com habilidades sociais e dificuldades de aprendizagem. Estudos de Psicologia (Natal), 11(2), 199-208.

Baraldi, D. M., \& Silvares, E. F. M. (2003). Treino de habilidades sociais e grupos com crianças agressivas, associado à orientação dos pais: Análise empírica de uma proposta de atendimento. In A. Del Prette \& Z. A. P. Del Prette (Eds.), Habilidades sociais, desenvolvimento e aprendizagem: Questões conceituais, avaliação e intervenção (pp. 235-258). Campinas, SP: Alínea.

Bolsoni-Silva, A. T. B., \& Marturano, E. D. (2002). Práticas educativas e problemas de comportamento: Uma análise à luz das habilidades sociais. Estudos de Psicologia (Natal), 7(2), 227-235.

Bolsoni-Silva, A. T., Marturano, E. M., Pereira, V. A., \& Manfrinato, J. W. S. (2006). Habilidades sociais e problemas de comportamento de pré-escolares: Comparando avaliações de mães e de professoras. Psicologia: Reflexão e Crítica, 19(3), 460-469.

Bordin, I. A. S., Mari, J. J., \& Caiero, M. F. (1995). Validação da versão brasileira do Child Behavior Checklist (CBCL): Inventário de comportamentos da infância e adolescência: dados preliminares: Revista ABP-APAL, 17(2), 55-66.

Caballo, V. E. (1996). O treinamento em habilidades sociais. In V. E. Caballo (Ed.), Manual de técnicas de terapia e modificação do comportamento (pp. 361-398). São Paulo, SP: Santos

Castro, R. E. F., Melo, M. H. S., \& Silvares, E. F. M. (2003). O julgamento de pares de crianças com dificuldades interativas após um modelo ampliado de intervenção. Psicologia: Reflexão e Crítica, 16, 309-318.

Coelho, M. V., \& Murta, S. G. (2007). Treinamento de pais em grupo: Um relato de experiência. Estudos de Psicologia (Campinas), 24(3), 333-341.

Del Prette, Z. A. P., \& Del Prette, A. (2001a). Psicologia das relações interpessoais. Vivências para o trabalho em grupo. Petrópolis, RJ: Vozes.

Del Prette, Z. A. P., \& Del Prette, A. (2001b). Habilidades sociais e educação: Pesquisa e atuação em Psicologia Escolar/Educacional. In Z. A. P. Del Prette (Ed.), Psicologia Escolar e Educacional: Saúde e qualidade de vida (pp. 113-141). Campinas, SP: Alínea.

Del Prette, Z. A. P., \& Del Prette, A. (2005). Psicología das habilidades sociais na infancia: Teoria e prática. Petrópolis, RJ: Vozes. 
Fariz, M. D., Mias, C. D., \& Moura, C. B. (2005). Comportamento agressivo e terapia cognitivo-comportamental na infância. In V. E. Caballo \& M. A. Simón (Eds.), Manual de Psicologia Clínica infantil e do adolescente (pp. 57-75). São Paulo, SP: Santos.

Gomide, P. I. C. (2003). Estilos parentais e comportamentos antisocial. In A. Del Prette \& Z. A. P. Del Prette (Eds.), Habilidades sociais, desenvolvimento e aprendizagem: Questões conceituais, avaliação e intervenção (pp. 21-60). Campinas, SP: Alínea.

Ladd, G. W., Herald, S. L., Slutzky, C. B., \& Andrews, R. K. (2004). Peer group rejection. In L. A. Rapp-Paglicci, C. N. Dulmus, \& J. S. Wodarski (Eds.), Handbook of preventive interventions for children and adolescents. (pp. 15-48). Hillsdale, NJ: John Willey \& Sons.

Löhr, S. S. (2003). Estimulando o desenvolvimento de habilidades sociais em idade escolar. In A. Del Prette \& Z. A. P. Del Prette (Eds.), Habilidades sociais, desenvolvimento e aprendizagem: Questões conceituais, avaliação e intervenção (pp. 293-310). Campinas, SP: Alínea.

Koch, L. M., \& Gross, A. M. (2005). Características clínicas e tratamento do transtorno da conduta. In V. E. Caballo \& M. A. Simón (Eds.), Manual de Psicologia Clínica infantil e do adolescente (pp. 23-38). São Paulo, SP: Santos.

Marinho, M. L. (2003). Comportamento anti-social infantil: Questões teóricas e de pesquisa. In A. Del Prette \& Z. A. P. Del Prette (Eds.), Habilidades sociais, desenvolvimento e aprendizagem: Questões conceituais, avaliação e intervenção (pp. 61-82). Campinas, SP: Alínea.

Marinho, M. L. (2005). Um programa estruturado para o treinamento dos pais. In V. E. Caballo \& M. A. Simón (Eds.), Manual de Psicologia Clínica infantil e do adolescente (pp. 417443). São Paulo, SP: Santos.

Marinho, M. L., \& Caballo, V. E. (2002). Comportamento antisocial infantil e seu impacto para a competência social. Psicologia, Saúde \& Doenças, 3(2), 141-147.

Moura, C. (2005). Será que conheço você? Jogo terapêutico para pais e filhos - Versão para crianças.

Murta, S.G. (2005). Aplicações do treinamento em habilidades sociais: Análise da produção nacional. Psicologia: Reflexão e Crítica, 18(2), 283-291.

Patterson, G., Reid, J., \& Dishion, T. (2002). Antisocial boys: Comportamento anti-social. Santo André, SP: Esetec.

Sánchez, A. V., \& Escribano, E. A. (1999). Medição do autoconceito. Bauru, SP: Edusc.

Stallard, P. (2004). Bons pensamentos - Bons sentimentos: Manual de terapia cognitivo- comportamental para crianças $e$ adolescentes. Porto Alegre, RS: Artmed. 